\title{
GAMeNT: A Framework to Formalize the Serious Game Design and Development
}

\author{
Aarón Yael Ponce-Guzmán, María Lucila Morales-Rodríguez, Claudia Gómez, \\ Nelson Rangel-Valdez, Laura Cruz-Reyes \\ TecNM/Instituto Tecnológico de Ciudad Madero, Tamaulipas, \\ Mexico \\ yaeloponce@gmail.com, lucila.morales@itcm.edu.mx,cggs71@hotmail.com, \\ rangel.nelson1980@gmail.com, cruzreyeslaura@gmail.com
}

\begin{abstract}
Games and video games entertain us and allow us to socialize and learn. Trying to take advantage of the interest in them and their characteristics as tools for education, the development of Serious Games has become popular. This paper presents the GAMeNT Framework which consists of an architecture and a methodology that guide the design and development of Serious Games. This framework was developed from a videogame framework, as a starting point, and it took into account the Serious Game inner elements to create the resultant architecture. The methodology was made after reviewing videogame's design processes and adapting them to include the Serious Games' elements.
\end{abstract}

Keywords: Serious games design, videogame framework, interactive storytelling, virtual agents.

\section{Introduction}

The games have always existed, they have helped us to entertain, to socialize, to learn, etc. This fact has remained since long ago. The Human being is vastly creative in the development of entertainment; one way is through the videogames, which are games that are played in electronic devices and that show us different experiences.

As part of improving said experiences, the storytelling it's a very important factor because it allows a better immersion in the game by making use of the virtual agents.

The videogames are an important part of the people; they represent an important business market that accounted for more than $\$ 99.3$ billion global revenues in 2016 [1]. Nowadays, there exist different areas of development in videogames, one of them, the Serious Games, tries to take advantage of the videogame's interest, characteristics, and technologies, in order to improve the education.

The Serious Games (SG) differ from the entertaining games in which instead of just fun, they also look for the provision of knowledge or the development of skills in those who play them [2]. This work defines one framework for the design and development of SG. The objective of the framework is to produce SG that provokes 
immersion of the players into the game; this could be achieved through the integration of Socio-Emotional Virtual Agents in an Interactive Storytelling.

\section{Related Work}

One of the difficulties that come up each time someone develops SG is the lack of a formal work that describes the internal elements to design and develop them. This occurs even though there exist in the literature several works that deal with the design and development of videogames. Section 2.1 describes the existing architectures, Section 2.2 summarizes the methodologies, and Section 2.3 revise some SG.

\subsection{Architectures for Videogames}

The work in [3] is one of the first designs proposed for videogames; it defines a game as relations among mechanics, dynamics, and aesthetics. Later in [4], the technology and story are incorporated as key parts in the game design.

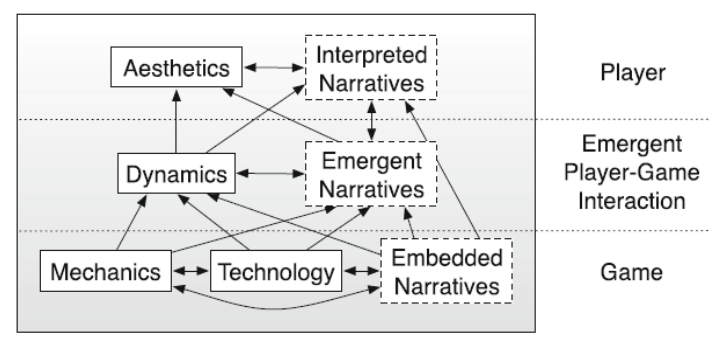

Fig. 1. Architecture of MTDA+N [5].

Table. 1. Description of MTDA+N architecture.

\begin{tabular}{l|l}
\hline \multicolumn{1}{c|}{ Components } & \multicolumn{1}{c}{ Definition } \\
\hline Mechanics & Describes data representation and algorithms of the game. \\
\hline Dynamics & $\begin{array}{l}\text { Describes the run-time behavior of the mechanics acting on the players' inputs to the } \\
\text { game and the results of this player interaction in the game over time. }\end{array}$ \\
\hline Aesthetics & Describes the desirable emotional responses evoked in the player. \\
\hline $\begin{array}{l}\text { Embedded } \\
\text { Narratives }\end{array}$ & They are the stories told by developers through narrative, mechanics, and gameplay. \\
\hline $\begin{array}{l}\text { Emergent } \\
\text { Narratives }\end{array}$ & They are a meaningful sequence of events that emerges during player-game interaction. \\
\hline $\begin{array}{l}\text { Interpreted } \\
\text { Narratives }\end{array}$ & $\begin{array}{l}\text { They are the mental representations of the player and the interpretations of the projected } \\
\text { or emergent narratives of a game. }\end{array}$ \\
\hline Technology & They are Tools and Systems used to implement or deliver the gameplay. \\
\hline
\end{tabular}

Finally, these previous works were integrated into the MTDA+N architecture proposed by [5] to develop videogames (see Figure 1). The main contribution of [5] is the definition of the design of a game through the three main layers, game, player, and player-game interaction. 
See Table 1 for the description of the components of MTDA+N's architecture. Note that this work divides the story of a game, included by [4], in three types of narratives.

\subsection{Methodologies for the Development of Videogames}

The revised architectures only provide information about the components of a videogame and their interactions. However, in order to develop a videogame, it is necessary to count with instructions that guide in this procedure. Normally, these instructions are organized in the form of methodologies. For example, the methodologies proposed in $[6,12]$ serves as guides from the game design to its implementation, these methodologies are shown in Table 2. Note that, for these methodologies, a game must have a goal clearly defined, and a theme that expresses that goal, i.e. the environment of the game.

Table 2. Methodologies of Game Design

\begin{tabular}{ll|l}
\hline \multicolumn{2}{c|}{ Crawford's game design sequence [6] } & \multicolumn{2}{|c}{ Fullerton's game design methodology [12] } \\
\hline 1. & Choose a goal and a theme & 1. Conceptualization \\
2. Research and preparation & 2. Prototyping \\
3. Design Phase & 3. Digital Prototyping \\
3.1 IO Structures & 4. Playtesting \\
$3.2 \quad$ Game Structure & 5. Functionality, Completeness, and Balance \\
$3.3 \quad$ Program Structure & 6. Fun and Accessibility \\
3.4 Evaluation of the design & & \\
4. Pre-programming Phase & & \\
5. Programming Phase & & \\
6. Playtesting Phase &
\end{tabular}

\subsection{Successful Serious Games}

There are different renowned serious games that are reported in the literature. In [711] are reported several of different learning areas that involve flight simulations, the solution of puzzles, army's life, among others. As videogames, these projects share common implementation elements as Static and Dynamic Objects, Events, and Nonplayer Characters (Virtual Agents), but they also share some others relative to SG, which are the inclusion of Narratives or Interactive Storytelling.

Summarizing, even though the work of [5] could be considered as a great advance in the definition of videogames, it lacks a formal methodology to develop them, as the ones proposed in $[6,12]$, which guide from the game design to its implementation. Also, the work of [5] is too general and does not facilitate the design of SG.

The present work presents the framework GAMeNT as an evolution of [5] towards the definition of SG. GAMeNT integrates learning content in the design to create such type of games, and it unifies both design and the methodology for its development. The following section details in depth the framework. 


\section{GAMeNT Framework}

The proposed SG framework is called GAMeNT. This name comes from the acronym of Spanish words Gameplay, Aprendizaje, Meta, Narrativas, and Tecnología. The words in Spanish stand for gameplay, learning, goal, narratives, and technologies, which represent the five most important modules that appear in its architecture for SG. As defined before, a SG needs to establish the learning content so, the GAMeNT includes it as the main module.

Being said that, this work evolves MTDA+N not only to design but also to develop SG. To do so, GAMeNT includes in its design the specification of the goal of what it is required to teach in-game, and it defines the synergy between this learning process and the elements involved in MTDA+N to reinforce the content to teach. Also, it guides on the construction process through a methodology.

The remaining of this section presents in Section 3.1 the architecture proposed by GAMeNT for the design of SG, in Section 3.2 the implementation elements derived from the architecture, and in Section 3.3 the methodology that guides through the implementation.

\subsection{GAMeNT Architecture Components}

The GAMeNT architecture is shown in Figure 2. It is formed, as commented previously, by the modules gameplay, learning, goal, narratives, and technologies. These modules are organized in the layers game, emergent player-game interaction, and player. The architecture reflects the synergy that allows the design of SG. The remaining of this section details the purpose of GAMeNT modules.

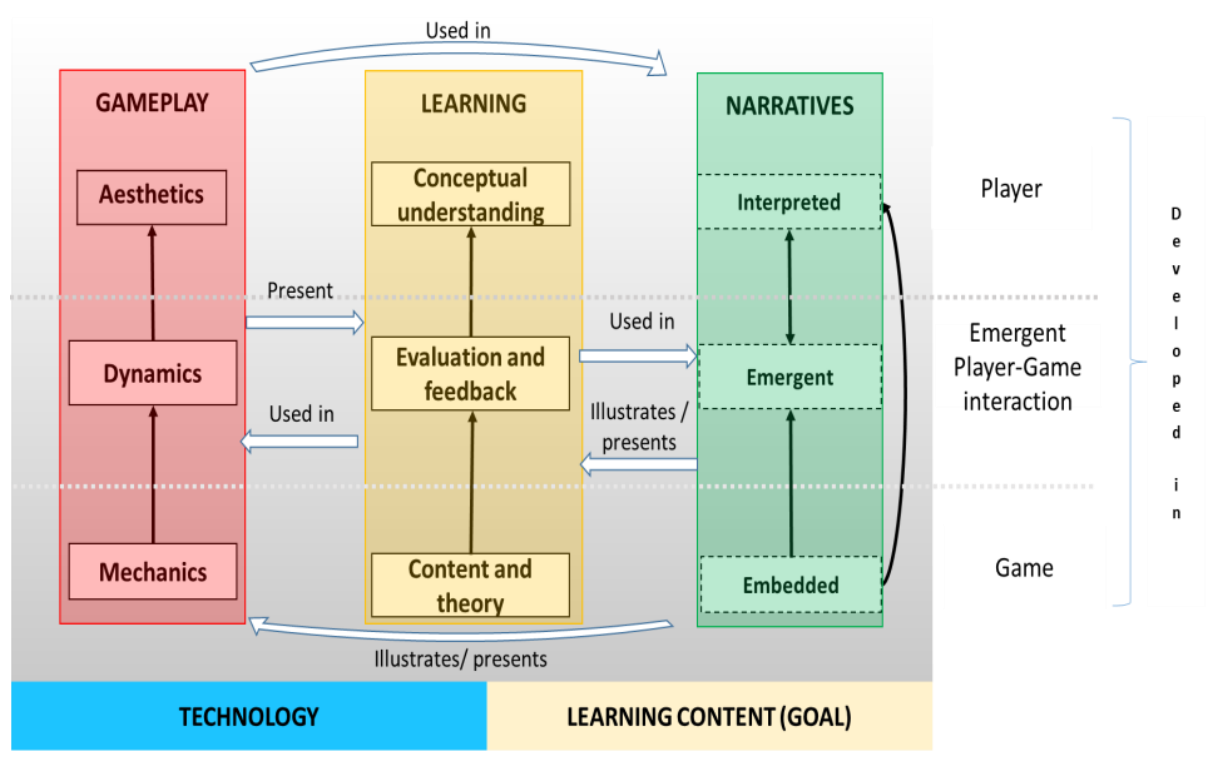

Fig. 1. Graphical Scheme of the GAMeNT architecture. 
Learning Content (Goal). This is the first base module that must be considered in the design of SG; it has as purpose to define the learning objective that will be pursued. Around this objective is defined the technology that should be used, and are building the gameplay, the learning, and the narratives.

Technology. This is the second base module for the design of SG. It leads the specification of technology required for gameplay, learning, and narratives. Mainly, it is used to define the scope and limitations in terms of interfaces, controls, game and develop platforms, graphic environment, language, etc.

Gameplay. This module group the Mechanics, Dynamics, and Aesthetics. GAMeNT uses the definitions of [3] shown above in Table 1, with a slight difference in Aesthetics, where not only are specified the emotional responses in the players, but also the visual and auditory aesthetics; this is done to aid in the creation of a better immersion.

Learning. This module explicitly defines the learning elements related to the learning objective pursued by the SG. The content and theory elements are the methods and/or methodologies to resolve a given problem. The evaluation and feedback elements are an essential part of the learning process, because they measure the actual state of the learner according to its actions or given answers; it also gives a feedback of its strong and weak points, how good were the answers, what areas need to be improved, and why [13]. The conceptual understanding element is developed in the player layer and it references to the understanding of the topic as a concept instead of the factual understanding. As said by [14], the conceptual understanding is more important than the factual understanding because the former one its deeper and harder to evaluate due to it requires the understanding of the topic, and not just memorize them.

Narratives. The Narratives module makes reference to the Storytelling inside the Serious Games, this module is used to present the Gameplay and the learning components in an enjoyable and entertaining way to the player, making a better immersion in the game through the story presented in the narratives.

\subsection{GAMeNT Implementation Elements}

As it was previously said in Section 2.3, a SG has as implementation elements Static and Dynamic Objects, Events, Virtual Agents, Narratives, and Interactive Storytelling. The interactions of these elements inside the GAMeNT architecture is shown in Figure 3; they are used to achieve a better cohesion among the modules, and are described as follows:

- The Static Objects let us have what doesn't change inside the game, this help to create the embedded narratives and the content and theory of the learning;

- The Events are used to show a content along the interaction of the player with the game environment through the emergent narratives; they also allow to create the way in which the player can be evaluated and given feedback; 


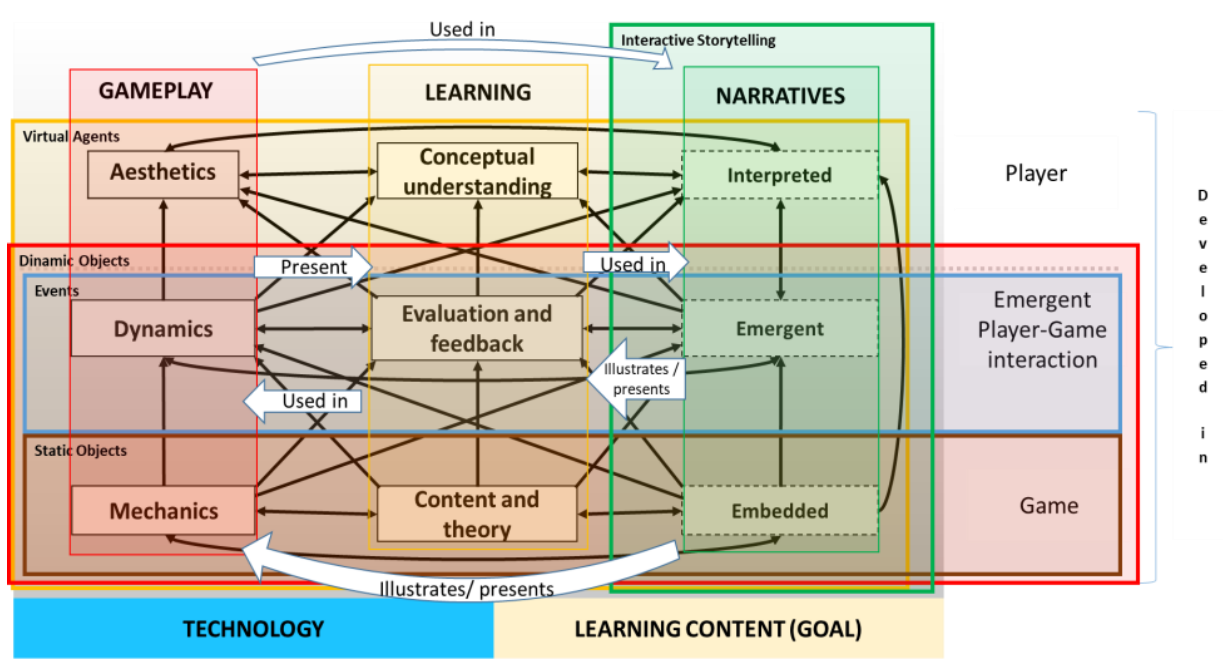

Fig. 3. Serious game elements in the GAMeNT architecture.

- The Dynamic Objects are elements with a defined behavior, and that will be used along the content and theory, the evaluation and feedback of the learning, and the embedded and emergent Narratives of the emergent Player-Game interactions; and

- The Virtual Agents (or NPC) are dynamic elements inside the game which are used to convey information to the player; it is expected that the NPC has an emotional response in the player, thus having defined Aesthetics. The NPCs serve to allow the realization of the embedded and emergent narratives, and the narratives interpreted by the player by the given socioemotional behavior. Using the agents, it will be easier the assimilation of information, and the conceptual understanding because they help in the creation of more realistic simulations.

\subsection{Methodology to Apply the GAMeNT Architecture}

In order to facilitate the use of the architecture, the framework GAMeNT integrates a methodology to design and develop SG. The steps of this methodology are as follows:

1. Establish the learning content (Goal) and the environment of the game (theme).

2. Research and prepare the learning content and the thematic:

(a) Review the state of art of the works that have been used the goal and theme selected to know how they have been approached and the qualities of them.

(b) Find and understand information about the learning content to prepare the theoretical content to present in the Serious Game.

(c) Research and understand the info about the theme to be allowed to design:

(i) The game environment to present in the Storytelling.

(ii) Environment Dynamics.

(iii) Environment Aesthetics. 
3. Create diverse ideas or concepts for the game to be used, improved or discarded as appropriate:

(a) Propose game environments and their visual and auditory elements that conform it.

(b) Establish: (i) Theoretical content, (ii) The Evaluation, (iii) The Feedback.

(c) Propose the Narrative idea developed in the game.

(d) Obtain level ideas to show in the game and their respective elements:

(i) Mechanics, (ii) Dynamics, (iii) Aesthetics, (iv) Narratives, (v) Learning Content

(e) Design the Virtual Agents:

(i) Define their Interactive Storytelling roll, (ii) Define their learning roll, (iii) Mechanics, (iv) Dynamics, (v) Aesthetics.

(f) Create events to show the learning content during the game levels:

(i) Propose their Dynamics, (ii) Propose their Aesthetics.

(g) Propose object whose will have interaction with the player and their elements:

(i) Mechanics, (ii) Dynamics, (iii) Aesthetics.

(h) Create elements for the player evaluation and feedback:

(i) Propose Dynamics and events for the evaluation, (ii) Propose Dynamics and events for the feedback.

4. Perform the design phase:

(a) IO Structure, (b) Game Structure, (c) Program Structure, (d) Evaluation of the design.

5. Create physical prototypes to corroborate that the mechanics perform their task.

6. Create digital prototypes to corroborate that the core system of the game is as desired/ Implementation of the game.

7. Playtesting.

8. Prove that the game is functional, internally complete and balanced.

9. Verify that the game still gives the desired experience and its usability.

This methodology was developed based on $[6,12]$ methodologies. These works are used as a complement of each other because while [6] makes emphasis in the design and preparation of the story, and the establishment of the learning content given it is a learning game, [12] focusses in the game design and its functionality.

In addition, the proposed methodology considers the implementation elements required in the design (see Section 3.2). In the methodology of GAMeNT, the steps 1 and 2 are focused on getting the necessary knowledge to design the SG. The steps 3 and 4 design the internal elements and the structures of the SG. The steps 5 and 6 are focused on development. Finally, the steps 7 to 9 are focused on the testing and verification of the desired experience, giving the same opportunities to their players.

\section{Conclusions and Future Work}

This paper has presented the GAMeNT framework that integrates design and development of Serious Game (SG) from the combination of architectures and 
methodologies. The main contribution lies in the formalization of the design and development of SG by explicitly indicating its core elements. With the GAMeNT framework, it is possible that newcomers can design a complete SG because they would only need to follow the methodology. It remains as future work to continue the exploration of alternatives that shifts from a conceptual model to a software framework and grants a set of elements to speed the implementation process; this represents the key component in future attempts to integrate the use of an established behavioral model for virtual agents, and tools that aid in the design and development of a wider variety of SG.

Acknowledgments. This work was partially supported by CONACyT, Universidad Autónoma de Nuevo León and TecNM/Instituto Tecnológico de Ciudad Madero. We particularly acknowledge the participation of Project 3058 from the Cátedras CONACyT program, Project 280081 from the program Redes Temáticas Conacyt and from Project 280712 of the Consolidation Program of National Laboratories.

\section{References}

1. Mobile, Esports \& Games Market Research, https://newzoo.com/insights/articles/ consumer-research -in-16-countries-covers-84-of-the-99-3bn-global-games-marketrevenues-in-2016/

2. Abt, C.C.: Serious Games. University Press of America (1987)

3. Hunicke, R., LeBlanc, M., Zubek, R.: MDA: A formal approach to game design and game research. In: Challenges in Game AI Workshop, AAAI Press, San Jose, CA (2004)

4. Schell, J.: The art of game design: a book of lenses. Elsevier/Morgan Kaufmann, Amsterdam, Boston (2008)

5. Ralph, P., Monu, K.: Toward a Unified Theory of Digital Games. Comput. Games J. 4, 81100 (2015)

6. Crawford, C.: The Art of Computer Game Design. Osborne/McGraw-Hill, Berkeley, CA, USA (1984)

7. America's Army, https://www.americasarmy.com/

8. A Force More Powerful, http://www.aforcemorepowerful.org/game/

9. ImpactGames: PeaceMaker: Play the News. Solve the Puzzle, http://www. peacemakergame.com/.

10. Center for Game Science: Solve Puzzles for Science, Foldit, https://fold.it/portal/.

11. Microsoft: Microsoft Flight Simulator, https://www.microsoft.com/Products/ Games/FSInsider/product/Pages/default.aspx

12. Fullerton, T., Swain, C., Hoffman, S.S.: Game Design Workshop-A playcentric approach to creating innovative games. Morgan Kaufmann Publishers (2008)

13. Searing, R.: Learning Through Games, But Not Gamification. Dice Tower News, http://www.dicetowernews.com/learning-through-games-but-not-gamification/15291

14. Berdik, C.: Make a Game out of Learning, http://www.slate.com/articles/technology/ future_tense/2015/04/mit_s_education_arcade_promotes_games_not_gamification_in_scho ols.html, (2015) 